\title{
CLIENTELA COM ALTERAÇÕES CARDÍACAS: PERFIL DIAGNÓSTICO ELABORADO POR ALUNOS DE ENFERMAGEM
}

\author{
Maria Márcia Bachion* \\ Maria Lúcia do Carmo Cruz Robazzi* \\ Emília Campos de Carvalho* \\ Eugênia Velludo Veiga*
}

\begin{abstract}
No presente estudo buscaram-se os padrões de respostas humanas e as categorias diagnósticas identificadas por 16 alunos de graduação em enfermagem junto a 22 pacientes portadores de alterações cardíacas, internados em um hospital escola. Foram encontrados 77 diagnósticos de enfermagem (D.E.), sendo 72,7\% do padrão TROCAR, 10,3\% do padrão CONHECER, 7,7\% do padrão MOVER, 6,4\% do padrão SENTIR e 2,5\% do padrão RELACIONAR. As categorias diagnósticas encontradas com maior freqüência foram: Déficit de Conhecimento (9\%); Débito Cardíaco Diminuído (7,7\%); Excesso de Volume de Líquido (6,4\%); Alteração na nutrição para menos que o corpo necessita $(6,4 \%)$ e Potencial para Infecção $(5,1 \%)$.
\end{abstract}

UNITERMOS: diagnóstico de enfermagem, ensino, alterações cardíacas

\section{INTRODUÇÃO}

As origens dos Diagnósticos de Enfermagem (D.E.) recaem em Florence Nightingale, embora o uso explícito deste termo seja atribuído a Veraa Fry, em $1953^{9}$.

*Docentes da Escola de Enfermagem de Ribeirão Preto da Universidade de São Paulo, Departamento de Enfermagem Geral e Especializada. Endereço para correspondência: Av. Bandeirantes, 3900. Ribeirão Preto, Estado de São Paulo. CEP: 14040-902 
$\mathrm{Na}$ década de 70, enfermeiras norte americanas passam a liderar um movimento global para sistematizar o emprego deste conceito e sua classificação. Constituíram, com esta finalidade, o Grupo Norte Americano para Classificação dos Diagnósticos de Enfermagem, que a partir de 1982 foi oficialmente transformado na North American Nursing Diagnosis Association (N.A.N.D.A.), com o intuito de desenvolver, aperfeiçoar e promover a taxonomia desses diagnósticos, para uso geral dos enfermeiros.

Em sua Nona Conferência realizada em 1990, a N.A.N.D.A. ${ }^{12}$ aprovou a seguinte definição para Diagnóstico de Enfermagem: "trata-se de um julgamento clínico das respostas do indivíduo, família ou da comunidade aos processos vitais ou aos problemas de saúde atuais ou potenciais, os quais fornecem a base para a seleção das intervenções de enfermagem para atingir resultados, pelos quais o enfermeiro é responsável".

Adotou ainda uma classificação para as possíveis reações ou respostas do ser humano; estas foram denominadas padrões e compõem a estrutura teórica para a organização dos diagnósticos. Os nove padrões são: TROCAR (liberar ou perder algum elemento, recebendo outro em retorno), COMUNICAR (transmitir interna ou externamente e de forma verbal ou não verbal, pensamentos, sentimentos e informações), RELACIONAR (estabelecer uma relação com outra coisa, pessoa ou lugar), VALORIZAR (estimativa de, conforme seu real ou suposto valor, utilidade ou importância; opinião do indivíduo sobre uma ligação a uma pessoa ou coisa), ESCOLHER (decidir em relação a um assunto onde a pessoa é livre agente e de acordo com suas inclinações), MOVER (mudar o corpo ou parte dele de posição, colocá-lo/conservá-lo em movimento), PERCEBER (tornar-se consciente de, através dos sentidos; captar o que não está visível ou presente para ser observado), CONHECER (estar familiarizado com, ser reconhecedor de, através de informação, relato, investigação, observação), SENTIR (experienciar a consciência, sensação, percepção ou significado, sendo afetado por um fato, evento ou estado) ${ }^{9}$.

Cada um destes padrões contempla um conjunto de possíveis alterações, com suas respectivas características definidoras (evidências) e fatores relacionados (etiologia). Ao formular um D.E., seguindo a taxonomia proposta pela N.A.N.D.A., o enfermeiro identifica o tipo de alteração (rótulo ou título), sua etiologia (quando conhecida) e as evidências obtidas na fase de coleta de dados. Tem-se como exemplo: comunicação verbal prejudicada (título de alteração) relacionada à diminuição da circulação cerebral (etiologia) evidenciada por dificuldade de formar frases e desorientação (evidências).

Apesar dos D.E. constituírem a base para a seleção de intervenções de Enfermagem, até o presente momento, ainda é incipiente o número de investigações que abordam essa temática em nosso país, ${ }^{4,5,6,7,9,11}$. Sabe-se que o ensino dos D.E., adotando a taxonomia da N.A.N.D.A., está sendo ministrado em algumas Escolas de Enfermagem brasileiras, em disciplinas isoladas, em nível de graduação e pósgraduação, não se tendo conhecimento, até a presente data, de um mapeamento 
das mesmas.

Ministrando este conteúdo a alunos do Curso de Graduação em Enfermagem, que freqüentam a disciplina Enfermagem Médica, na Escola de Enfermagem de Ribeirão Preto da Universidade de São Paulo, as autoras desse estudo visualizaram como principal objetivo identificar os padrões e as categorias diagnósticas, atribuídos pelos alunos de enfermagem a pacientes com alterações cardíacas.

\section{METODOLOGIA}

Após freqüentarem aulas teóricas sobre D.E. e "Assistência de Enfermagem à pacientes com Alterações Cardíacas", dentre outros temas previstos na disciplina Enfermagem Médica, os 66 alunos do $5^{\circ}$ semestre do Curso de Graduação em Enfermagem, no ano de 1992, foram subdivididos em 8 grupos, por ordem alfabética, para realizarem atividades práticas em um hospital escola, utilizado como campo de estágio supervisionado nessa disciplina.

Dois destes grupos, totalizando $16(24,2 \%)$ alunos tiveram sob seus cuidados 22 pacientes, cujos Diagnósticos Médicos pressupunham alterações cardíacas. A relação aluno/número de pacientes variou em decorrência da rotatividade dos pacientes e da gravidade de seus estados, no decorrer de duas semanas de atividades práticas desenvolvidas junto às enfermarias de cardiologia do mencionado hospital.

Aos alunos foi solicitado que desenvolvessem as atividades assistenciais adotando método específico composto das fases: coleta de dados, elaboração de D.E. (empregando-se a Taxonomia I da NANDA), planejamento (meta, objetivo e prescrição), implementação e avaliação. dos D.E.

Neste estudo, os dados descritos reportam-se apenas à fase de elaboração

\section{RESULTADOS E DISCUSSÃO}

Foram elaborados 77 D.E. pelos graduandos de enfermagem aos 22 pacientes assistidos. Observou-se a variação de 1 (um) a 6 (seis) D.E. estabelecidos para cada paciente, com o predomínio da freqüência de 4 (quatro) diagnósticos por cliente. Estas informações estão demonstradas na Figura 1. 


\section{FIGURA 1 - FREQÜÊNCIA DE DIAGNÓSTICOS DE ENFERMAGEM PARA CADA PACIENTE}

Número de Diagnósticos de Enfermagem

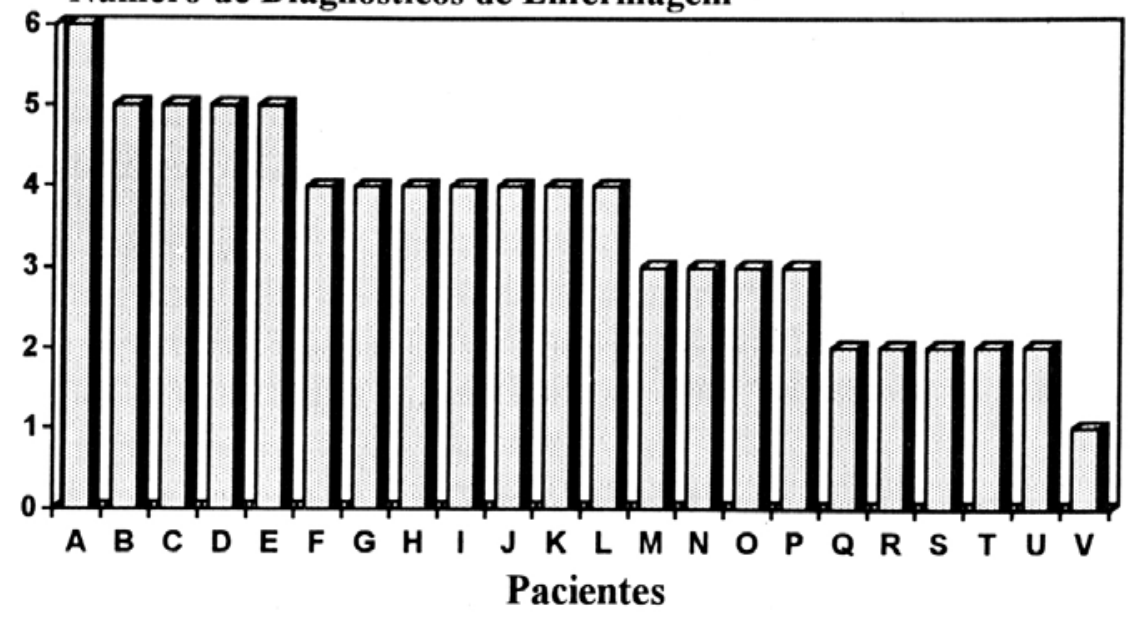

Quanto aos padrões de respostas humanas, verificou-se que a grande maioria dos D.E. atribuídos pelos estudantes aos pacientes encontra-se no padrão TROCAR (72,7\%), seguidos dos inseridos no padrão CONHECER (10,38\%), MOVER (7,79\%), SENTIR $(6,49 \%)$ e RELACIONAR (2,59\%). Os padrões COMUNICAR, VALORIZAR, ESCOLHER e PERCEBER não foram contemplados na atribuição diagnóstica.

Este achado sugere que os alunos têm sua atenção predominantemente voltada para as alterações biológicas manifestadas pela clientela assistida. Tal fato pode ser justificado visto serem tais alterações mais facilmente identificadas, i que, por sua vez, pode estar sendo reforçado pelo modelo de ensino adotado na disciplina de Enfermagem Médica, na Escola, como um todo, bem como pela prática hospitalar vigente.

A avaliação de outras alterações, que não as físicas mostrou-se limitada, desde o momento da concepção mental de quais aspectos deveriam ser observados no cliente, considerando-se que os próprios alunos, naquele ano, sugeriram e elaboraram o instrumento de coleta de dados, que iriam utilizar no decorrer da disciplina. Para uma abordagem global, acredita-se que é necessário sistematizar a busca de dados abrangendo diferentes aspectos, caso contrário, dificilmente serão estabelecidos diagnósticos em padrões tais como VALORIZAR e ESCOLHER.

Essa dificuldade de avaliar outras áreas, que não a biológica, também pode ser evidenciada em publicações ${ }^{1,2,8,11,13}$ que têm explorado predominantemente alterações dessa natureza.

Os D.E. encontrados em cada um dos padrões estão descritos na Tabela 1, a seguir.

Os D.E. mais freqüentes no padrão TROCAR foram: Débito Cardíaco Diminuído e Alteração na Mucosa Oral, cada um aparecendo com a freqüência de 6 (7,79\%). O primeiro diagnóstico é esperado em pacientes com patologias cardíacas, devido ao comprometimento dos mecanismos reguladores que ocorre nestes casos. Quanto ao segundo, dadas as características sócio-econômicas culturais dos pacientes, foi encontrado em decorrência de higiene oral inefetiva, além da presença de dentes cariados. 
TABELA 1 - FREQÜÊNCIA DE CATEGORIAS DIAGNÓSTICAS ENCONTRADAS PELOS ALUNOS JUNTO AOS PACIENTES

TABELAI - Frequência de categorias diagnósticas encontradas pelos alunos junto aos pacientes

\begin{tabular}{|c|c|c|c|c|c|c|c|c|c|c|c|c|c|c|c|c|c|c|c|c|c|c|c|c|}
\hline Padråa/Categoria Diagnóstica & & $P$ & $\bar{A}$ & $\bar{c}$ & $\bar{T}$ & $\bar{E}$ & $\bar{N}$ & 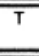 & $\bar{E}$ & $\bar{s}$ & & & & & & & & & & & & & & TAL \\
\hline & 1 & 2 & 3 & 4 & 5 & 6 & 7 & 8 & 9 & 10 & 11 & 12 & 13 & 14 & 15 & 16 & 17 & 18 & 19 & 20 & 21 & 22 & & $\overline{\%}$ \\
\hline $\begin{array}{l}\text { TROCAR } \\
\text { Excesso de volume liquido }\end{array}$ & $\mathrm{x}$ & & & & & $\mathrm{x}$ & & & & & & & & $\bar{x}$ & $\mathrm{x}$ & & & & & $x$ & & & 5 & 6.49 \\
\hline Integridade da pele prejudicada & $\mathrm{x}$ & $\mathrm{x}$ & & & & & & $\mathrm{x}$ & & & & & & & & & & & & & & & 3 & 3,89 \\
\hline Alteraçào na mucosa oral & & $\mathrm{x}$ & & $\mathrm{x}$ & $\mathrm{x}$ & $\mathrm{x}$ & & & & $x$ & $x$ & & & & & & & & & & & & 6 & 7.79 \\
\hline Débito cardiaco diminuido & & & $x$ & & & & & $x$ & & & $x$ & & & & $\mathrm{x}$ & & & $x$ & & & $x$ & & 6 & 7,79 \\
\hline $\begin{array}{l}\text { Alteraç̧ào da Nutrição menos do } \\
\text { que o corpo necessita }\end{array}$ & & & & $\mathrm{x}$ & $x$ & & & & & & & & & $x$ & & & $x$ & $x$ & & & & & 5 & 6,49 \\
\hline Alteraçăo na perfusão tissular & & & & & & & & & \begin{tabular}{|lll}
$x$ & \\
\end{tabular} & & & & & & & & & & & & & & 1 & 1,29 \\
\hline $\begin{array}{l}\text { Incapacidade de desobstruir } \\
\text { vias aéreas }\end{array}$ & & & & & & & & & & & $\mathrm{x}$ & & & & & & $x$ & & $\mathrm{x}$ & & & & 3 & 3,89 \\
\hline Integridade tissular prejudicada & & & & & $\mathrm{x}$ & & & & & & $x$ & & & & & & & & & & & & 2 & 2,59 \\
\hline Padrāo respiratório ineficaz & & & & & & & & & & & & $\mathrm{x}$ & & & & $\bar{x}$ & & & & & & & 2 & 2,59 \\
\hline Troca de gases prejudicada & & & & & & & & & & & & & & $x$ & & & & & E & - & 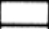 & & 1 & 1,29 \\
\hline Retençào urinária & 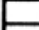 & & & & & & & & & & & & & & & & & & & & 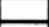 & $\mathrm{x}$ & 1 & 1.29 \\
\hline $\begin{array}{l}\text { POTENCIAL } \\
\text { Para excesso de volume liquido }\end{array}$ & & & $\mathrm{x}$ & & & & & $\mathrm{x}$ & & & & & & & & $\mathrm{x}$ & & & & & & & 3 & 3,89 \\
\hline Para ferimentos & $\mathrm{x}$ & & $x$ & & & & $x$ & & $\mathrm{x}$ & & & & & & & & & & & & & & 5 & 6,49 \\
\hline $\begin{array}{l}\text { Para alteraçào na nutriça me- } \\
\text { nos do que o corpo necessita }\end{array}$ & & & $x$ & & & & & & & & & & & & & & & & & & & & 1 & 1.29 \\
\hline $\begin{array}{l}\text { Para déficit do volume de } \\
\text { liquido }\end{array}$ & & & & & $\mathrm{x}$ & & & & & & & & & & & & & & & & & & 1 & 1.29 \\
\hline Para infeç̧ăo & & & & & & $\mathrm{x}$ & $\mathrm{x}$ & & & & & & $x$ & & & & & & & & & $\bar{x}$ & 4 & 5,19 \\
\hline $\begin{array}{l}\text { Para prejuizo na integridade da } \\
\text { pele }\end{array}$ & & & & & & $\mathrm{x}$ & & & & & & & & & & & & & & & & & 1 & 1.29 \\
\hline $\begin{array}{l}\text { Para alteraçăo da temperatura } \\
\text { corporal }\end{array}$ & & & & & & & & & & & & & & & & & $x$ & & & & & & 1 & 1,29 \\
\hline Para sindrome de desuso & & & & & & & & & & & & & & & & & & & $x$ & & & & 1 & 1.29 \\
\hline $\begin{array}{l}\text { Para diminuiçào do débito } \\
\text { cardiaco }\end{array}$ & & & & & & & $\mathrm{x}$ & & & & & & & & & & & & $x$ & & & $x$ & 2 & 2,59 \\
\hline Para constipaçăo intestinal & & & & & & & & & & & & & & & & & & & $\mathrm{x}$ & & & & 1 & 1.29 \\
\hline $\begin{array}{l}\text { Para prejuizo na integridade da } \\
\text { pele }\end{array}$ & & & & & & & & & & & & & & & & & & & & $x$ & & & 1 & 1.29 \\
\hline $\begin{array}{l}\text { RELACIONAR } \\
\text { Isolamento social }\end{array}$ & & & & $x$ & & & & & & & & & & & & & & & & & & & 1 & 1.29 \\
\hline Difunçăo sexual & & & & & & & & & & & & & $\mathrm{x}$ & & & & & & & & & & 1 & 1,29 \\
\hline $\begin{array}{l}\text { MOVER } \\
\text { Distúrbio no padrào do sono }\end{array}$ & & & & $x$ & & $x$ & & & & & & & & & & & & & & & & & 2 & 2.59 \\
\hline $\begin{array}{l}\text { Déficit para o auto cuidado } \\
\text { (higiene corporal, higiene oral) }\end{array}$ & & & & & & & & $x$ & & & & & & & & & & & & & & & 1 & 1.29 \\
\hline Intolerância à atividade fisica & & & & & & & & & $\mathrm{x}$ & $\mathrm{x}$ & & & & $x$ & & & & & & & & & 3 & 3.89 \\
\hline $\begin{array}{l}\text { CONHECER } \\
\text { Déficit de conhecimento }\end{array}$ & \begin{tabular}{|l|}
$x$ \\
$x$ \\
\end{tabular} & $x$ & & & & & & & & & & & & $x$ & $\bar{x}$ & $x$ & $x$ & & & & & & 7 & 9.00 \\
\hline $\begin{array}{l}\text { Alleraçào no processo de } \\
\text { pensamento }\end{array}$ & & & & & $\mathrm{x}$ & & & & & & & & & & & & & & & & & & 1 & 1.29 \\
\hline $\begin{array}{l}\text { SENTIR } \\
\text { Dor crônica }\end{array}$ & & & & & & & & & & & & & $x$ & & & & & & & & & & 1 & 1,29 \\
\hline Dor & & $x$ & & & $\mathrm{x}$ & & & & & & & & & & & & & & & & & & 2 & 2,59 \\
\hline Ansiedade & & & & & & & & & \begin{tabular}{|lll}
$x$ & & $r$
\end{tabular} & & & & & & & & & & & & & & 1 & 1,29 \\
\hline Medo & & & & & & & & & & & & $x$ & & & & & & & & & & & 1 & 1.29 \\
\hline TOTAL & 5 & 4 & 4 & 4 & 6 & 5 & 3 & 4 & 4 & 2 & 4 & 2 & 3 & 5 & 2 & 2 & 5 & 3 & 4 & 2 & 1 & 3 & & $100 \%$ \\
\hline
\end{tabular}


Observou-se ainda a presença dos D.E.: Excesso de Volume de Líquido; Alteração na Nutrição menos que o corpo necessita e Potencial para Ferimentos, tendo cada um deles a freqüência de $5(6,49 \%)$. O primeiro aparece provavelmente em conseqüência das alterações dos mecanismos reguladores; o segundo relacionado à diminuição da ingestão alimentar decorrente da cronicidade da patologia ou das características da dieta preconizada para alguns casos ou ainda, devido à inabilidade dos pacientes graves em levarem o alimento até a boca. O terceiro diagnóstico pode ser decorrente, predominantemente, de distúrbios metabólicos, imobilidade no leito e hipóxia tecidual.

A presença de D.E. Potencial para Infecção (5,19\%), pode ser justificada pela estase de líquidos corporais e procedimentos invasivos a que esses pacientes estão sujeitos.

No estudo ficou evidenciada a necessidade de atenção à função respiratória uma vez que os D.E. atribuídos pelos alunos, nesta área foram: Incapacidade de Desobstruir Vias Aéreas (3,89\%), Padrão Respiratório Ineficaz (2,59\%), Troca de Gases Prejudicada (1,29\%), ligados à congestão venosa.

Foram constatados também os diagnósticos: Integridade de Pele Prejudicada (3,89\%), Integridade Tissular Prejudicada (2,59\%) e Alteração na Perfusão Tissular $(1,29 \%)$ pelo fato destes clientes serem submetidos à procedimentos invasivos e apresentarem mobilidade diminuída e integridade do músculo cardíaco comprometida.

Destacam-se, no presente estudo, dificuldades no desenvolvimento do julgamento clínico de determinadas respostas humanas, como por exemplo, Potencial para Débito Cardíaco Diminuído e Potencial para Excesso de Volume de Líquido. Considerando-se as interfaces de ambos e a sua validação até o momento, pela N.A.N.D.A., esses D.E. indubitavelmente merecem ser assunto de pesquisas futuras na enfermagem, a exemplo do estudo de DALTON ${ }^{8}$.

Percebe-se que as alterações no padrão TROCAR, devem-se provavelmente ao comprometimento do mecanismo de bombeamento cardíaco, que causa impacto em outros sistemas e funções.

Quanto ao padrão CONHECER, foram atribuídos 7 D.E. (9,0\%) de Déficit de Conhecimento, predominantemente relacionados à falta de exposição (orientação) relatada pelos pacientes sobre sua doença, ação de medicamentos que utilizam e exames complementares realizados. Infere-se aqui a provável ineficácia ou ineficiência das estratégias empregadas nas orientações ao invés de sua ausência, como informam os pacientes. Teve-se ainda neste padrão atribuição de 1 (um) diagnóstico de Alteração no Processo de Pensamento, relacionado à alteração metabólica, por disfunção renal.

No trabalho de GUIMARÃES \& VIANNA ${ }^{11}$, foram avaliados 23 pacientes portadores de valvulopatias reumáticas, quanto à presença dos D.E. de Excesso de Volume de Líquido e de Déficit de Conhecimento. Esses autores constataram alteração do primeiro tipo em $56 \%$ dos pacientes e Déficit de Conhecimento em $91 \%$ dos casos. 
Infere-se que os clientes que participaram do presente estudo foram subavaliados, pelos alunos, quanto à categoria diagnóstica de Déficit de conhecimento. Reforça esta inferência os achados de VEIGA et $\mathrm{al}^{14}$, que mostram o desconhecimento (acima de 50\%) da população da região de Ribeirão Preto-SP acerca de fatores de risco para doenças cardíacas (estresse, uso excessivo de sal, contraceptivos hormonais, estilo de vida sedentário, ingestão excessiva de álcool, dieta hipercalórica, tabagismo e hereditariedade). Estes resultados sugerem a necessidade de se organizar e implementar programas de educação continuada, que contemplem os das condições de saúde dos portadores de alterações cardiovasculares.

Os D.E. relatados no padrão MOVER foram: Intolerância à Atividade Física $(3,89 \%)$ relacionado à desequilíbrio entre demanda e suprimento de oxigênio, bem como necessidade de repouso no leito; Distúrbio no Padrão de Sono $(2,59 \%)$ relacionado à mudança de ambiente; Déficit para o Auto-cuidado: banho e higiene oral $(1,29 \%)$, relacionado à intolerância à atividade física. Novamente observa-se que os achados relacionam-se ao distúrbio cardíaco, o qual leva à restrição da mobilidade e atividade física do indivíduo.

Referente ao padrão SENTIR foram observados os D.E., de Dor (2,59\%), Dor Crônica (1,29\%), Ansiedade (1,29\%) e Medo (1,29\%). Os dois primeiros reportam-se a agentes de injúria biológica (alterações coronarianas) e os últimos foram evidenciados por verbalização de apreensão à situação vivida e medo frente aos exames propostos para elucidação dos Diagnósticos Médicos.

Quanto ao padrão RELACIONAR, foram observados os D.E., de Isolamento Social $(1,29 \%)$ relacionado à inabilidade para engajar-se em relacionamento pessoal satisfatório e alteração na aparência física; Disfunção Sexual $(1,29 \%)$ relacionado à falta de pessoa significativa (companheiro). Acredita-se ter havido também uma subavaliação dos aspectos ligados à sexualidade, pois, como se sabe, é significativa a preocupação nesta área entre os indivíduos portadores de problemas cardíacos, principalmente os que sofreram infarto do miocárdio.

OLIVEIRA JÚNIOR ${ }^{13}$ alerta que pouca atenção tem sido dispensada pelos médicos a esta temática e reforça a necessidade da sexualidade ser encarada com a devida relevância, como parte do conjunto das sensações humanas, ao nível físico e emocional. GARCIA ${ }^{10}$ evidencia a importância do preparo do enfermeiro nesta área.

Como pode ser percebido, alguns D.E. relacionam-se mais estreitamente às causas da própria internação (alterações cardíacas). Este fato reitera a necessidade da coleta de dados ser realizada de forma ampla, possibilitando uma assistência de enfermagem global nos diferentes padrões de respostas humanas.

\section{CONCLUSÕES E RECOMENDAÇÕES}

*Os $16(24,2 \%$ do total de 66$)$ graduandos de enfermagem do $5^{\circ}$ semestre do Curso de Graduação em Enfermagem identificaram 77 D.E., durante o período 
de duas semanas de atividades de assistência a 22 pacientes com alterações cardíacas. Houve predomínio da freqüência de 4 D.E. por cliente;

*a grande maioria dos D.E. atribuídos encontra-se no padrão TROCAR (72,7\%), seguidos dos que estão no padrão CONHECER (10,38\%), MOVER (7,79\%), SENTIR (6,49\%) e RELACIONAR (2,59\%). Os padrões COMUNICAR, VALORIZAR, e PERCEBER não foram contemplados na atribuição dos D.E., pelos estudantes;

*evidenciou-se uma predominância de D.E. relacionados às alterações biológicas, achado esse concordante com a literatura, que também tem explorado predominantemente, alterações dessa natureza.

\section{CONSIDERAÇÕES FINAIS}

* Provavelmente o modelo de ensino adotado na disciplina Enfermagem Médica e nas demais disciplinas anteriores a essa, na grade curricular da Escola como um todo, tem reforçado os aspectos biológicos no atendimento ao paciente, em detrimento de outros igualmente importantes de serem considerados na formação de futuros enfermeiros;

* As estratégias de ensino devem contemplar o paciente em seus diversos aspectos (biológicos, emocional e social) e possibilitar a identificação de dados alterados em outros padrões de respostas humanas, além dos que forem identificados neste estudo.

\section{CLIENTELE WITH CARDIAC ALTERATIONS: A DIAGNOSIS PROFILE ELABORATED BY NURSING STUDENTS}

The present study aimed at searching patterns of human responses and diagnoses categories identified by 16 undergraduate nursing students in 22 patients with cardiac alterations admitted in a School Hospital. 77 Nursing Diagnoses (ND) were found, $72,7 \%$ in the pattern of EXCHANGING, $7,7 \%$ in the pattern of MOVING, $6,4 \%$ in the pattern of FEELING and 2,5\% in the pattern of RELATING. The predominant diagnoses categories were: knowledge deficit (9\%), decreased cardiac output $(7,7 \%)$, fluid volume excess (6,4\%), altered nutrition less than body requirements $(6,4 \%)$ and high risk for infection $(5,1 \%)$.

UNITERMS: nursing diagnosis, teaching, cardiac alterations 


\section{CLIENTELA CON ALTERACIONES CARDÍACAS: PERFIL DIAGNÓSTICO ELABORADO POR ALUMNOS DE ENFERMERÍA}

En el presente estudio los autores buscaron los patrones de respuestas humanas y las categorías diagnósticas identificadas por 16 alumnos de graduación en enfermería en 22 pacientes con alteraciones cardíacas, internados en un Hospital Escuela. 77 Diagnósticos de Enfermería fueron encontrados, $72,7 \%$ en el patrón INTERCAMBIO, 10,3\% en el patrón CONOCER, 7,7\% en el patrón MOVILIZAR, 6,4\% en el patrón SENTIR y 2,5\% en el patrón RELACIONAR. Las categorías diagnósticas encontradas con más frecuencia fueron: Déficit de Conocimiento (9\%), Débito Cardíaco disminuido (7,7\%), Exceso de Volumen de Líquido (6,4\%), Alteración de la Nutrición, menos de las necesidades del cuerpo $(6,4 \%)$ y Potencial para Infección $(5,1 \%)$.

UNITERMOS: diagnósticos de enfermería, enseñanza, alteraciones cardíacas

\section{REFERÊNCIAS BIBLIOGRÁFICAS}

01. ALMEIDA, F.A. et al. Extra-sístoles ventriculares: condutas de enfermagem. Rev. Paul. Enfermagem, São Paulo, v. 9, n. 2, p. 65-72, mai/ago, 1990.

02. BARBATO, M.G. et al. Principais problemas fisiológicos apresentados por pacientes com infarto do miocárdio e as respectivas prescrições de enfermagem. Rev. Esc. Enfermagem, São Paulo, v. 13, n. 2, p. 147-55, 1979.

03. CARVALHO, E. C. Diagnósticos do padrão de resposta humana COMUNICAR. In: SIMPÓSIO NACIONAL SOBRE DIAGNÓSTICOS DE ENFERMAGEM, 3, João Pessoa, Pb., 1992. Anais. João Pessoa, 1992. p. 105-13.

04. CARVALHO, E.C.; BACHION, M.M.; BERNARDINA, L.D. O processo diagnóstico em enfermagem: aplicação em paciente submetido a transplante renal. Ribeirão Preto. Escola de Enfermagem de Ribeirão Preto - USP. 1993. p. 23. (Mimeografado)

05. CARVALHO, E.C. et al. Elaboração de diagnósticos de enfermagem a pacientes com alterações cardíacas: dificuldades de alunos de enfermagem. Ribeirão Preto. Escola de Enfermagem de Ribeirão Preto. 1993. p. 7. (Mimeografado)

06. CARVALHO, E.C. et al. The process of diagnosis and its teaching. Ribeirão Preto. Escola de Enfermagem de Ribeirão Preto. 1994. p. 13. (Mimeografado) 
07. CRUZ, I.C.F. Diagnóstico de enfermagem: estratégia para sua formulação e validação. São Paulo, p. 195. Tese (Doutorado) - Escola de Enfermagem, Universidade de São Paulo, 1993.

08. DALTON, J. A descriptive study: defining characteristics of the nursing diagnosis cardiac output, alterations in: decreased. Image., v. 27, n. 4, p. 113-17, 1985.

09. FARIAS, J.N. et al. Diagnóstico de enfermagem: uma abordagem conceitual e prática. João Pessoa, PB: Santa Marta, 1990. p. 160.

10. GARCIA, T.R. Sexualidade humana: conhecimento necessário à formação do enfermeiro. Acta Paul. Enfermagem, São Paulo, v. 6, n. 1/4, p. 39-42. jan/dez, 1993.

11. GUIMARÃES, H.C.Q.C.P., VIANNA, L.A.C. Características do paciente valvulopata com diagnóstico de excesso de volume de líquido e déficit de conhecimento sobre a doença. Rev.Soc.Cardiol.Est.São Paulo, v. 4, p. 58. 1993. Supl. A.

12. NORTH AMERICAN NURSING DIAGNOSIS ASSOCIATION. Taxonomy I revised 1990: with official diagnostic categories. St. Louis, 1990.

13. OLIVEIRA JÚNIOR, W. Atividade sexual após o infarto do miocárdio. Arq.Bras.Cardiol., v. 46, n. 3, p. 205-10, mar. 1986.

14. VEIGA, E.V. et al. Estudo dos fatores de risco da hipertensão arterial: conhecimento e exposição. Rev.Soc.Cardiol.Est.São Paulo, v. 3, n. 6, p. 15, nov/dez. 1993. Supl. A. 